\title{
Nanoscale tribology of graphene grown by chemical vapor deposition and transferred onto silicon oxide substrates
}

\author{
Tuna Demirbaş and Mehmet Z. Baykara ${ }^{a)}$ \\ Department of Mechanical Engineering and UNAM - Institute of Materials Science and Nanotechnology, \\ Bilkent University, Ankara 06800, Turkey
}

(Received 27 November 2015; accepted 30 December 2015)

\begin{abstract}
We present a comprehensive nanoscale tribological characterization of single-layer graphene grown by chemical vapor deposition (CVD) and transferred onto silicon oxide $\left(\mathrm{SiO}_{2}\right)$ substrates. Specifically, the nanotribological properties of graphene samples are studied via atomic force microscopy (AFM) under ambient conditions using calibrated probes, by measuring the evolution of friction force with increasing normal load. The effect of using different probes and post-transfer cleaning procedures on frictional behavior is evaluated. A new method of quantifying lubrication performance based on measured friction coefficient ratios of graphene and $\mathrm{SiO}_{2}$ is introduced. A comparison of lubrication properties with mechanically-exfoliated graphene is performed. Results indicate that CVD-grown graphene constitutes a very good solid lubricant on $\mathrm{SiO}_{2}$, reducing friction coefficients by $\sim 90 \%$ for all investigated samples. Finally, the effect of wrinkles associated with CVD-grown graphene on measured friction values is quantitatively analyzed, with results revealing a substantial increase in friction on these structural defects.
\end{abstract}

\section{INTRODUCTION}

To extend the lifespan of mechanical systems, wear and consequently, friction must be minimized. Liquidphase lubricants featuring a variety of additives are typically used toward this end in macroscopic mechanical systems. On the other hand, traditional liquid-based lubrication schemes fail in nano- and micro-scale systems comprising mobile components due to increasing surfaceto-volume ratios and the associated enhancement in physical effects such as surface tension. ${ }^{1,2}$ As such, research efforts in recent years have been aimed at identifying candidates for solid lubricants to be used in nano- and micro-scale mechanical systems and characterizing related structural and tribological properties.

Among various candidates for solid lubricants, the two-dimensional material graphene is of particular importance not only due to its outstanding electrical ${ }^{3}$ and mechanical ${ }^{4}$ properties, but also because it constitutes the essential building block of bulk graphite, a widely-used solid lubricant in a variety of macroscopic applications. ${ }^{5}$ Consequently, based on its promise as a single-atom-thick solid lubricant consisting of a sheet of carbon atoms arranged in a honeycomb pattern, the nanotribological properties of graphene have been investigated via several studies in the literature. ${ }^{6-13}$

\footnotetext{
Contributing Editor: Mohd Fadzli Bin Abdollah

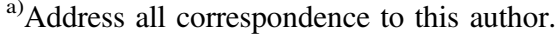

e-mail: mehmet.baykara@bilkent.edu.tr

DOI: $10.1557 / \mathrm{jmr} .2016 .11$
}

In particular, nanotribological characterization of singleand multi-layer graphene samples obtained via mechanical exfoliation has revealed a layer-dependent frictional behavior, with the friction values observed via atomic force microscopy (AFM) decreasing monotonously with increasing number of layers. ${ }^{8}$ This finding has been explained by the so-called puckering effect that involves the enhanced build-up of graphene in front of the scanning probe at small number of layers (owing to reduced out-of-plane stiffness), which leads to increased friction. On the other hand, for graphene samples grown epitaxially on $\mathrm{SiC}$, friction was also measured via $\mathrm{AFM}$, and it was found that bi-layer graphene demonstrated lower friction than both single-layer graphene and bulk graphite samples. ${ }^{6}$ These findings have been partially explained by an electron-phonon coupling mechanism.

While the nanotribological properties of mechanicallyexfoliated and epitaxially-grown graphene samples are of great interest from a fundamental point of view, limitations associated with sample size (for mechanicallyexfoliated graphene) and transfer possibilities onto different substrates (for epitaxially-grown graphene) limit the related application potential. On the other hand, high quality single- and multi-layer graphene of sufficient dimensions for practical applications can be grown on metal substrates, such as nickel and copper, via the method of chemical vapor deposition (CVD), ${ }^{14,15}$ which is typically followed by transfer onto substrates such as silicon oxide $\left(\mathrm{SiO}_{2}\right)$ for further characterization of, e.g., electrical and mechanical properties. When it comes to the characterization of nanotribological properties of 
CVD-grown graphene, there are only a limited number of studies available in the literature: Egberts et al. have studied the nanotribological properties CVD-grown graphene on copper foils (i.e., pre-transfer), with the main discovery that friction forces on graphene patches exhibit hysteretic behavior with changing normal load. ${ }^{12}$ On the other hand, Fessler et al. have studied the effect of plasma-hydrogenation on nanotribological behavior of CVD-grown graphene on $\mathrm{SiO}_{2}$ substrates. ${ }^{11}$ Moreover, Kim et al. used a probe in the shape of a fused silica lens to investigate the friction and adhesion properties of CVD-grown graphene transferred onto $\mathrm{SiO}_{2}$ substrates. ${ }^{9}$ Very recently, Paolicelli et al. have studied the frictional properties of graphene grown by CVD on $\mathrm{Ni}(111)$ substrates and contrasted the results with mechanicallyexfoliated graphene samples on $\mathrm{SiO}_{2}{ }^{13}$

Motivated by the discussion above, we present in this article a comprehensive structural and nanotribological characterization of single-layer, CVD-grown graphene transferred onto $\mathrm{SiO}_{2}$ substrates. Graphene films are grown on copper foils via CVD of methane $\left(\mathrm{CH}_{4}\right)$ and subsequently transferred onto $\mathrm{SiO}_{2}$ substrates. Structural and morphological properties of graphene samples are characterized using optical microscopy, scanning electron microscopy (SEM), Raman spectroscopy, and AFM. The nanotribological properties of CVD-grown graphene samples transferred onto $\mathrm{SiO}_{2}$ substrates are studied via AFM under ambient conditions using calibrated probes, by recording the evolution of friction force with increasing normal load. In particular, and in contrast to the available literature, the potential influence of using (i) different probes and (ii) post-transfer cleaning procedures on nanotribological behavior is investigated. Additionally, a new method of analyzing lubrication performance aimed at eliminating the role of the probe on nanotribological measurements that involves the evaluation of the ratio of friction coefficients of graphene and $\mathrm{SiO}_{2}$ is introduced. Lastly, a comparison of lubrication performance of CVD-grown graphene with mechanically-exfoliated graphene is presented and the effect of wrinkles on measured friction values is quantitatively examined.

\section{EXPERIMENTAL DETAILS}

\section{A. Sample preparation}

\section{Graphene growth on copper foils via CVD}

Copper and nickel foils are widely used as catalysts for the CVD growth of graphene. Due to significant differences in the solubility of carbon in the two materials $(0.001 \%$ for copper versus $0.1 \%$ for nickel), the growth mechanism differentiates for copper and nickel substrates such that graphene grown on nickel features a large amount of multilayer regions, ${ }^{14}$ whereas CVD-growth on copper performed by the deposition of $\mathrm{CH}_{4}$ at elevated temperatures $\left(\sim 1000{ }^{\circ} \mathrm{C}\right)$ results in almost uniform coverage of the substrate by single-layer graphene. ${ }^{15}$ As the aim of the present study is to characterize the nanotribological properties of single-layer graphene samples, CVD growth on copper has been preferred. Specifically, $25 \mu \mathrm{m}$ thick copper foil with $99.8 \%$ purity (Alfa Aesar 13382, Lancashire, United Kingdom) has been chosen, in accordance with the literature. ${ }^{15}$ The foil has been cut in dimensions of $35 \mathrm{~mm} \times 50 \mathrm{~mm}$, cleaned via subsequent baths in acetone and isopropanol, dipped into a diluted chloric acid solution $(10 \mathrm{~mL} \mathrm{HCl}, 90 \mathrm{~mL}$ water) to remove surface oxide, and cleaned again via distilled water. The cut and cleaned copper foil pieces are then placed into the quartz tube furnace (Alser Teknik/ProTherm, Ankara, Turkey) used for the CVD process. At the start of the CVD process, the copper foils were annealed at $1000{ }^{\circ} \mathrm{C}$ under a reducing flow of $\mathrm{Ar} / \mathrm{H}_{2}$ gas mixture (95:5) at $200 \mathrm{sccm}$ for $30 \mathrm{~min}$ to remove any remaining copper oxide and to increase the average grain size. After thermally annealing the copper foil, graphene growth was initiated with the introduction of $\mathrm{CH}_{4}$ into the quartz tube at a typical flow rate of $25 \mathrm{sccm}$ for $20 \mathrm{~min}$. The heating was then turned off, and the graphene-covered copper foil was taken out of the furnace after cooldown to room temperature. Photographs showing the change in the appearance of a representative copper foil after CVD growth of graphene are provided in Fig. 1, together with a high-resolution optical microscopy image of the graphene-covered copper foil surface that demonstrates the conformal coverage of graphene on the topographical features of the foil.

\section{Transfer of CVD-grown graphene onto $\mathrm{SiO}_{2}$ substrates}

As the next step in sample preparation, graphene films grown via CVD on copper foils have been transferred onto $\mathrm{SiO}_{2}$ substrates, for eventual structural and nanotribological characterization. Toward this purpose, a sacrificial carrier layer of poly(methyl methacrylate) (PMMA) has been utilized, in accordance with established procedures in the literature. ${ }^{15}$ The whole transfer procedure took place in a class 100 cleanroom. In particular, during the transfer procedure, (i) one side of the graphene-covered copper foil is spin-coated with PMMA (950K A2), (ii) the PMMA is cured at $110{ }^{\circ} \mathrm{C}$ for $15 \mathrm{~s}$ on a hot plate, (iii) a diluted nitric acid solution ( $5 \mathrm{~mL} \mathrm{HNO}_{3}, 15 \mathrm{~mL}$ water) is used to etch away the graphene on the side of the copper foil that is not covered by PMMA, and (iv) a $1 \mathrm{M}$ solution of ammonium persulfate is utilized at $70{ }^{\circ} \mathrm{C}$ to etch away the copper foil, leaving behind a strip of PMMA covered with CVDgrown graphene on one side. Subsequently, the PMMA strip is placed on a clean $\mathrm{SiO}_{2}$ wafer piece, and the PMMA is removed via dipping the sample into acetone. The individual steps of the transfer procedure are summarized in Fig. 2. 

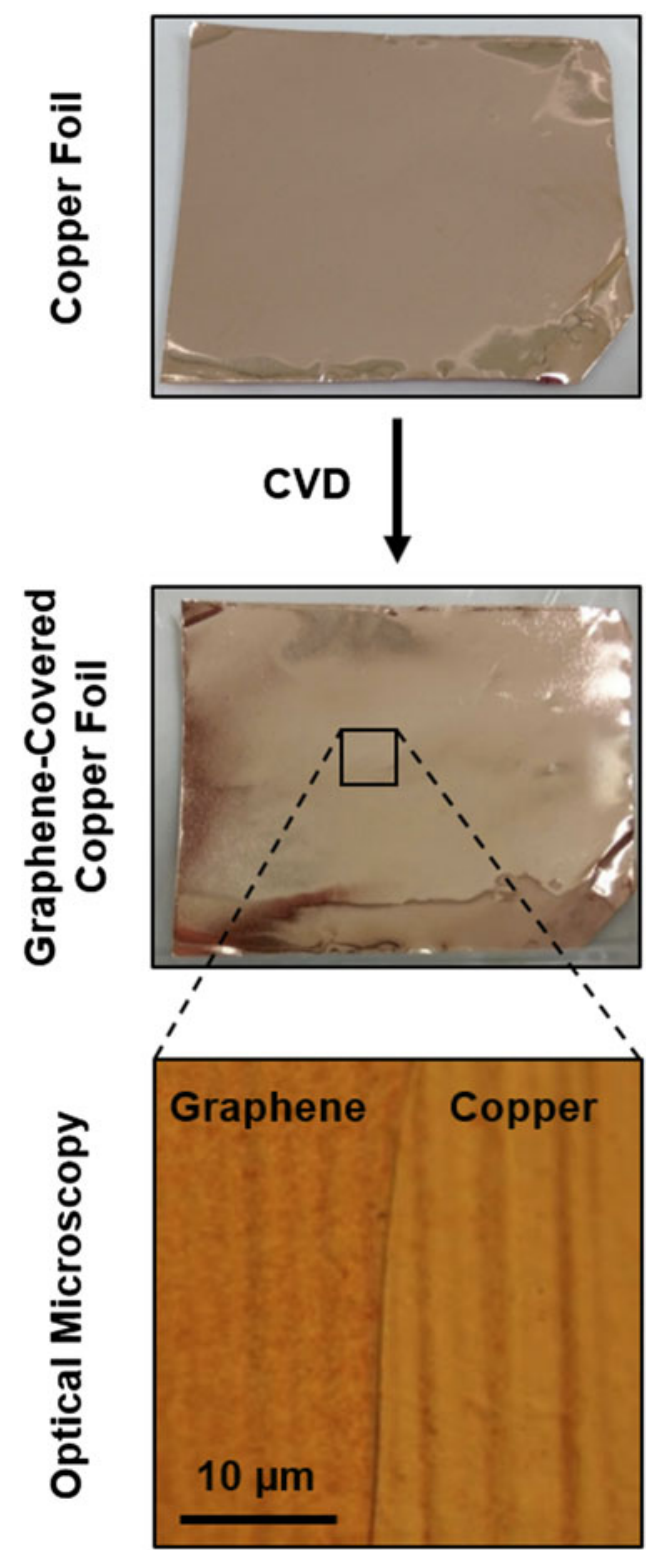

FIG. 1. Photographs of a copper foil before and after CVD-growth of graphene, together with an optical microscopy image of a partially graphene covered region.

\section{Post-transfer cleaning of CVD-grown graphene}

Placing the PMMA-graphene stack in a still bath of acetone is often not sufficient to remove the whole PMMA layer from graphene-residues are commonly observed, even with the resolution provided by optical microscopy. To remove PMMA residues, various strategies have been suggested in the literature, ranging from using glacial acetic acid as a solvent, ${ }^{16}$ to thermal annealing under vacuum, ${ }^{17}$ as well as in the presence of gases such as $\mathrm{Ar},{ }^{18} \mathrm{Ar} / \mathrm{H}_{2},{ }^{19} \mathrm{CO}_{2},{ }^{20}$ and $\mathrm{N}_{2}{ }^{21}$ Despite intensive efforts involving high temperatures and extended annealing times, it is typically observed that PMMA residues are never entirely removed from the graphene surface. ${ }^{22}$ To evaluate the potential influence of post-transfer cleaning on the nanotribological properties of CVD-grown graphene, two different methods of cleaning have been utilized in the present study: (i) short (20 s) ultrasonic baths of acetone followed by isopropanol and alternatively, (ii) annealing at $300{ }^{\circ} \mathrm{C}$ for $2 \mathrm{~h}$ under Ar gas flow in a quartz tube furnace.

\section{B. Structural characterization}

Before a comprehensive nanotribological characterization of CVD-grown graphene on $\mathrm{SiO}_{2}$ substrates is performed, the structural/morphological quality of the samples needs to be investigated and the single-layer character needs to be confirmed. Accordingly, in this work, optical microscopy (Carl Zeiss Axio Imager.A2m, Thornwood, New York) has been used to confirm the overall coverage of graphene on copper foils and $\mathrm{SiO}_{2}$, SEM (FEI Quanta 200 FEG, Hillsboro, Oregon, typically operated at $10 \mathrm{kV}$ ) has been used to microscopically analyze the presence of structural defects including tears and folds, and Raman spectroscopy (WITec Alpha300 S, Ulm, Germany, equipped with a $532 \mathrm{~nm}$ solid-state laser) has been utilized to confirm the single-layer character of graphene samples. ${ }^{23}$ Lastly, AFM (PSIA XE-100, Suwon, Korea) in contact-mode has been used for detailed topographical characterization to (i) independently check single-layer character by measuring the height of graphene on $\mathrm{SiO}_{2}$, (ii) detect the potential existence of contaminants, such as PMMA residues, and (iii) image structural defects such as wrinkles that are not straightforward to resolve using other methods such as SEM.

\section{Nanotribological characterization}

To measure the nanotribological properties of graphene, the AFM instrument has been operated in the socalled friction force microscopy mode which allows the recording of the lateral forces experienced by the probe apex during contact-mode scanning of the sample surface. $^{24}$ The measurements have been performed under ambient conditions and a total of five silicon probes (Nanosensors PPP-CONTR, Neuchatel, Switzerland) have been utilized as force sensors. To precisely detect the normal and lateral forces experienced by the probes, normal spring constant $(k)$ and lateral force calibration factor $(\alpha)$ values have been determined for each probe via calibration procedures described by Sader et al. ${ }^{25}$ and Varenberg et al., ${ }^{26}$ respectively (Table I). The half-width of friction loops formed by lateral forces in the forward and backward scanning directions has been used to determine friction force $\left(F_{\mathrm{f}}\right)$ values. ${ }^{27}$ In particular, the determination of the lateral force calibration factor $\alpha$ (that relates the voltage output of the AFM instrument due to the change in the horizontal position of the laser spot on the four-quadrant photodiode caused by the torsional 


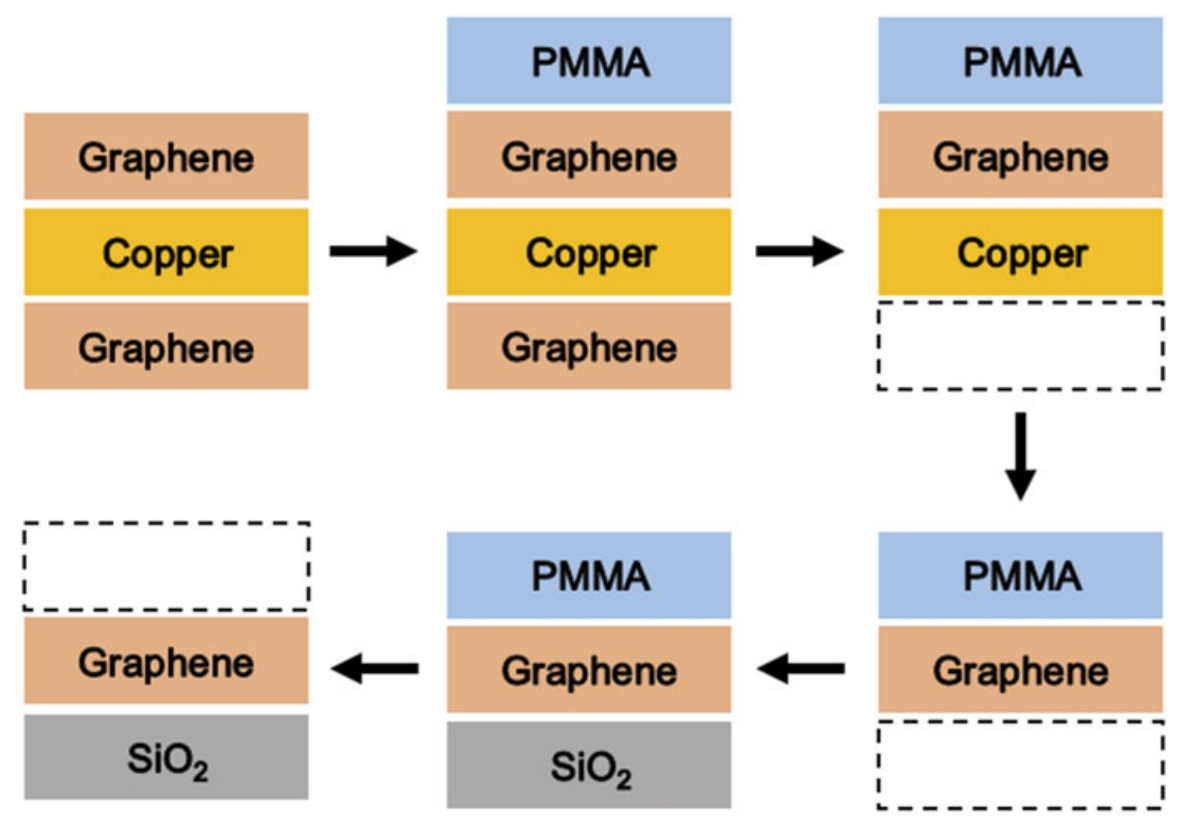

FIG. 2. The process flow associated with the transfer of CVD-grown graphene onto $\mathrm{SiO}_{2}$, as described in detail in the main text.

TABLE I. Normal spring constants $(k)$ and lateral force calibration factors $(\alpha)$ of AFM probes used for nanotribological characterization.

\begin{tabular}{lcc}
\hline \hline Probe number & $\begin{array}{c}\text { Normal spring } \\
\text { constant }(\mathrm{N} / \mathrm{m})\end{array}$ & $\begin{array}{c}\text { Lateral force calibration } \\
\text { factor }(\mathrm{nN} / \mathrm{V})\end{array}$ \\
\hline 1 & $0.24 \pm 0.03$ & $10.00 \pm 1.25$ \\
2 & $0.16 \pm 0.02$ & $15.00 \pm 2.00$ \\
3 & $0.24 \pm 0.03$ & $12.00 \pm 1.50$ \\
4 & $0.15 \pm 0.02$ & $19.00 \pm 2.50$ \\
5 & $0.13 \pm 0.02$ & $30.00 \pm 3.75$ \\
\hline
\end{tabular}

twisting of the cantilever to the lateral forces acting on the probe) involves the calculation of the half-width and offset of friction loops recorded on the sloped faces of a commercial calibration grating (MikroMasch TGF11, Sofia, Bulgaria) as a function of normal load and the utilization of force equilibrium arguments (for more details, see Ref. 26).

\section{RESULTS AND DISCUSSION}

\section{A. Structural properties of CVD-grown graphene on $\mathrm{SiO}_{2}$ substrates}

\section{Structural investigation via optical microscopy and SEM}

Single-layer graphene samples can be detected via optical microscopy when placed on silicon wafers covered with $\sim 300 \mathrm{~nm}$ thick $\mathrm{SiO}_{2} .{ }^{3}$ Consequently, optical microscopy has been used as the first step in the present study to detect and confirm the presence of CVD-grown graphene on $\mathrm{SiO}_{2}$ substrates post-transfer. While optical microscopy readily confirms large-scale coverage (millimeters and above) of $\mathrm{SiO}_{2}$ substrates by transferred graphene films, the spatial resolution is not sufficient to detect the presence of all structural defects such as tears and folds that frequently occur during transfer. As such, the next step in structural characterization of graphene films has involved imaging via SEM. Figure 3(a) shows the general appearance of CVD-grown graphene transferred onto $\mathrm{SiO}_{2}$ substrates via a large-scale SEM image that independently confirms the successful transfer of extended graphene films onto $\mathrm{SiO}_{2}$ substrates. Moreover, SEM images with higher resolution are utilized to investigate the presence of structural defects and contaminants in the transferred graphene films. For instance, Fig. 3(b) shows the morphology associated with a tear and additionally demonstrates the presence of contaminants in the form of small white dots on graphene samples that are occasionally encountered. While determining the exact chemical nature and origin of these contaminants is beyond the scope of the present study, energy dispersive $x$-ray spectroscopy indicates that oxygen is involved, supporting the idea that the white dots comprise copper oxide that originates from the foils used for CVD growth. ${ }^{28}$

\section{Confirmation of single-layer character via Raman spectroscopy}

Raman spectroscopy is frequently used to characterize the structure and chemistry of single- and multi-layer graphene. ${ }^{23}$ Specifically, the number of layers of graphene samples can be precisely determined by detecting the relative intensities of the characteristic $2 \mathrm{D}$ and $\mathrm{G}$ peaks $\left(I_{2 \mathrm{D} / \mathrm{G}}\right)$, such that $I_{2 \mathrm{D} / \mathrm{G}}$ values of $\geq 2, \sim 1$, and $<1$, are expected for single-layer, bi-layer, and multi-layer graphene samples, respectively. Figure 4 presents 


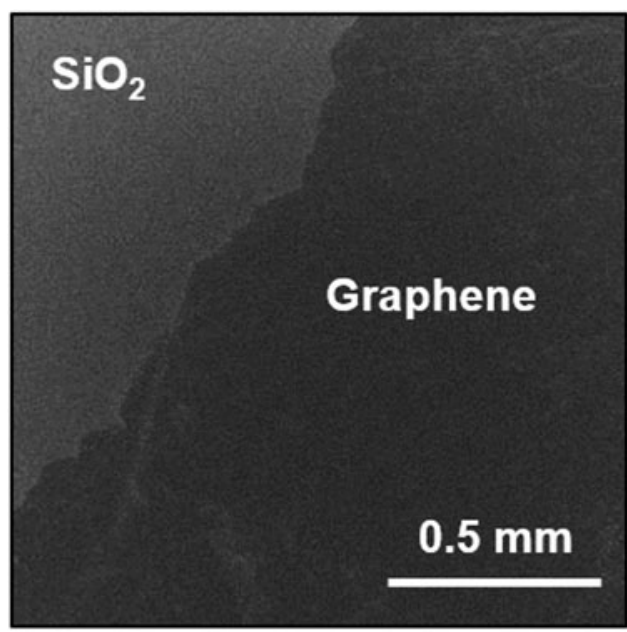

(a)

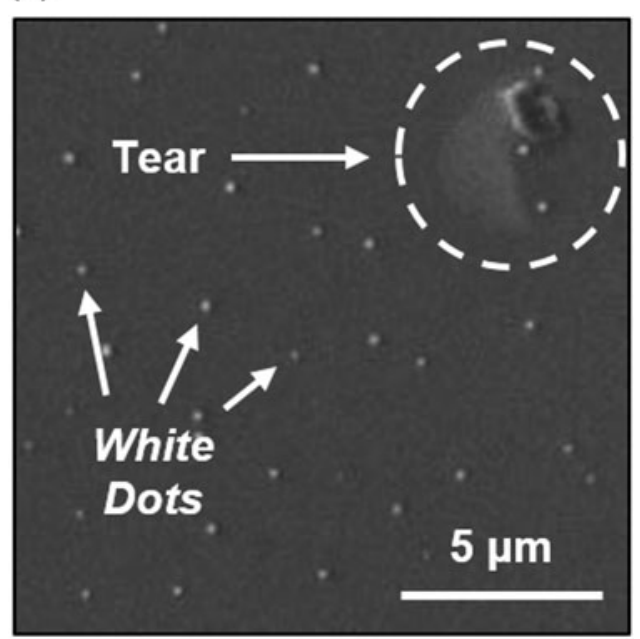

(b)

FIG. 3. (a) Large-scale SEM image confirming the transfer of extended graphene films onto $\mathrm{SiO}_{2}$. (b) Higher-resolution SEM image showing a tear in the transferred film as well as the occasional presence of contaminants in the form of white dots.

a typical Raman spectrum obtained on a CVD-grown graphene sample transferred onto $\mathrm{SiO}_{2}$. While the $I_{2 \mathrm{D} / \mathrm{G}}$ value of 2.80 is clearly indicative of the single-layer character, it should be mentioned that there is considerable variability in measured $I_{2 \mathrm{D} / \mathrm{G}}$ values between different graphene samples, and even for spectra obtained on the same graphene sample at different locations such that $I_{2 \mathrm{D} / \mathrm{G}}$ values between 2.50 and up to 5.50 have been recorded for the graphene films investigated in the present study. The observation of $I_{2 \mathrm{D} / \mathrm{G}}$ values significantly higher than 3.00 can be tentatively attributed to the presence of partially-suspended regions of transferred graphene films on $\mathrm{SiO}_{2} \cdot{ }^{29}$

\section{Structural investigation via AFM}

As already mentioned, AFM is particularly useful for graphene research in detecting the topographical height

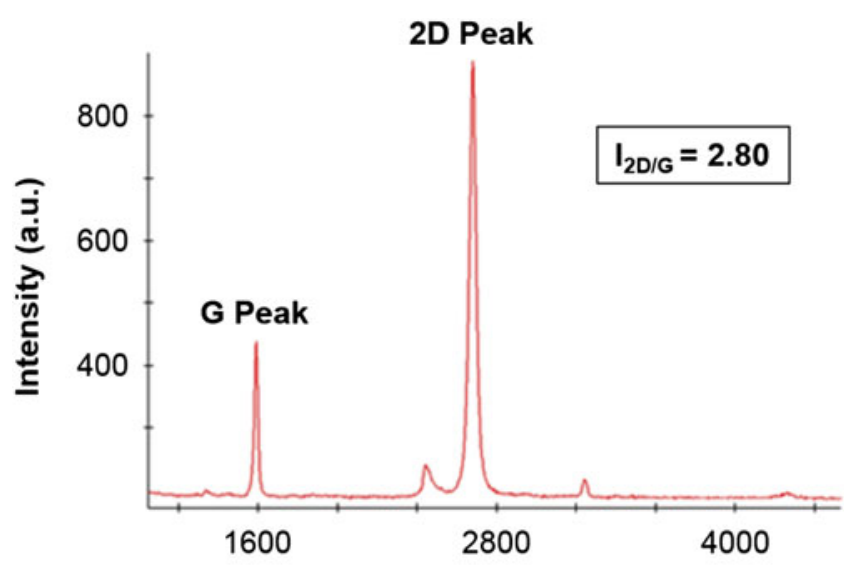

Raman Shift $\left(\mathrm{cm}^{-1}\right)$

FIG. 4. Representative Raman spectrum of a CVD-grown graphene sample transferred onto $\mathrm{SiO}_{2}$.

of graphene samples on $\mathrm{SiO}_{2}$ and in observing structural defects and chemical contaminants with high resolution. In particular, thanks to the high spatial resolution of the method, AFM can be readily used to detect wrinkles on CVD-grown graphene samples that arise during the cooldown step of the CVD process due to the substantial difference in the thermal expansion coefficients of graphene and copper, leading to a build-up of in-plane stress in graphene and consequently, wrinkling. ${ }^{15}$

A representative topographical AFM image of a region of the $\mathrm{SiO}_{2}$ substrate partially covered by graphene is provided in Fig. 5(a). It is observed that the graphene film features a number of structural defects including folds, and a substantial number of wrinkles. Moreover, the presence of contaminants (potentially, PMMA residues) on the graphene film is detected, which leads to occasional streaks in the AFM image due to the probe laterally manipulating the residues. Clearly, the high spatial resolution provided by the AFM method allows the investigation of graphene morphology and cleanliness in much higher detail when compared to optical microscopy and SEM. Figures 5(b) and 5(c) show topographical height profiles over the graphene- $\mathrm{SiO}_{2}$ boundary and over a representative wrinkle, respectively. The height of the transferred graphene with respect to the $\mathrm{SiO}_{2}$ substrate is measured as $\sim 1.45 \mathrm{~nm}$, in line with certain values reported in the literature. ${ }^{30}$ Moreover, the height of a representative wrinkle with respect to the graphene surface is observed as $\sim 2.80 \mathrm{~nm}$. A statistical analysis of wrinkle heights conducted via AFM on transferred graphene samples provides a mean value of $1.99 \mathrm{~nm}$ with a standard deviation (SD) of $0.87 \mathrm{~nm}$. Finally, it should be indicated that no substantial change in the morphology and improvement in the cleanliness of transferred graphene samples is detected via AFM after both variants of post-transfer cleaning, confirming reports in the literature that currently used methods aiming at removing PMMA residues are not entirely successful. ${ }^{22}$ 


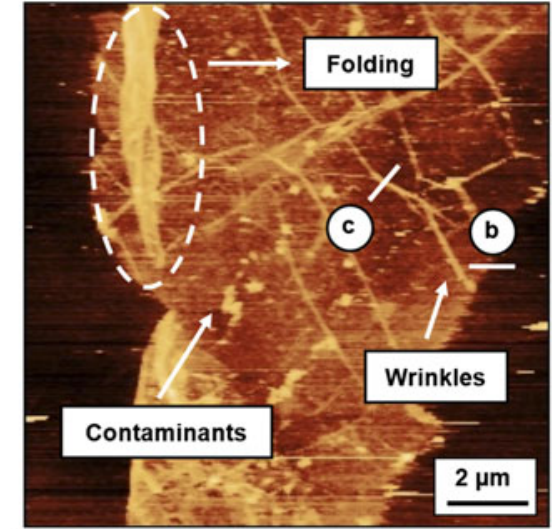

(a)
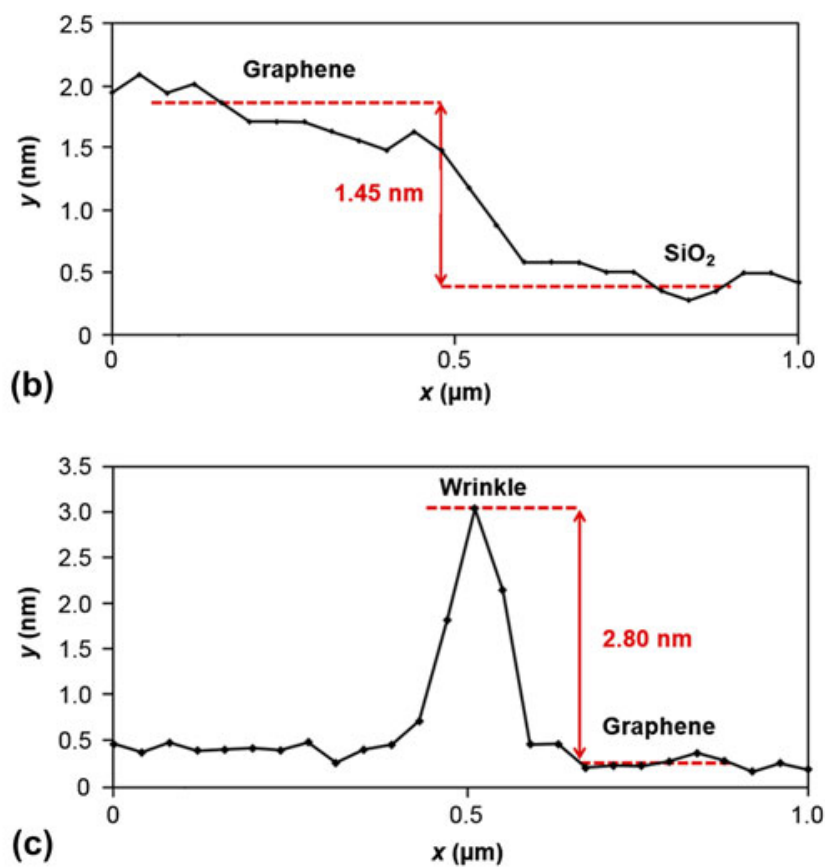

FIG. 5. (a) Topographical AFM image of CVD-grown graphene on $\mathrm{SiO}_{2}$, demonstrating in high resolution the presence of defects such as a fold and wrinkles on the graphene surface, as well as contaminants. (b) Representative height profile of the boundary between graphene and $\mathrm{SiO}_{2}$. (c) Representative height profile of a wrinkle on graphene. The height profiles in (b) and (c) have been recorded along the white lines designated with the letters "b" and "c" in (a), respectively.

\section{B. Nanotribological properties of CVD-grown graphene on $\mathrm{SiO}_{2}$ substrates}

1. Dependence of friction forces measured on CVD-grown graphene, wrinkles, and the $\mathrm{SiO}_{2}$ substrate on normal load

Friction forces $\left(F_{\mathrm{f}}\right)$ recorded on CVD-grown graphene, increased friction forces encountered at wrinkles, as well as friction forces recorded on the $\mathrm{SiO}_{2}$ substrate have been investigated as a function of normal load $\left(F_{n}\right)$ in the present study. A representative friction force map and corresponding results of $F_{\mathrm{f}}$ versus $F_{\mathrm{n}}$ measurements acquired with a single AFM probe are provided in Fig. 6. The $\mathrm{SiO}_{2}$ substrate exhibits the highest absolute $F_{\mathrm{f}}$ values, followed by wrinkles and CVD-grown graphene. The lubrication properties of CVD-grown graphene on the $\mathrm{SiO}_{2}$ substrate become apparent, as a significant reduction in absolute $F_{\mathrm{f}}$ values. The increase in $F_{\mathrm{f}}$ values measured on wrinkles is attributed to the associated abrupt change in topography, in accordance with the so-called ratchet effect. ${ }^{31}$ Please note that this is in contrast to previous observations of reduced friction on wrinkles of CVD-grown graphene patches on $\mathrm{Cu}$ foils which were attributed to multilayer character arising from a "fold-over" geometry at the wrinkle edges. ${ }^{12}$

It is observed that the data in Fig. 6(b) can be fit reasonably well in a linear fashion. To eliminate the effect of potentially different degrees of adhesion exhibited by the $\mathrm{SiO}_{2}$ substrate when compared to CVD-grown graphene on measured $F_{\mathrm{f}}$ values, tribological properties may be quantified by the slopes of linear fits applied to the data, to determine friction coefficients $(\mu)$. While the data presented in Fig. 6(b) already demonstrates that not only absolute $F_{\mathrm{f}}$ values, but also $\mu$ values are significantly lower on CVD-grown graphene when compared to $\mathrm{SiO}_{2}\left(\mu_{\text {Graphene }}: 0.006 \pm\right.$ $0.001, \mu_{\text {Wrinkle }}: 0.018 \pm 0.002$, and $\mu_{\mathrm{SiO}_{2}}: 0.072 \pm 0.003$ ); a related, comprehensive analysis of lubrication properties of CVD-grown graphene is presented in Sec. III. B. 5.

\section{Effect of using different probes on friction}

It is well known that the specific nanoscale structure and chemistry of AFM probes affect measured friction force values significantly. ${ }^{32}$ As such, several different probes have been used to perform the friction force measurements presented here. The results of $F_{\mathrm{f}}$ versus $F_{\mathrm{n}}$ experiments conducted on CVD-grown graphene with three different probes (probes 1-3) are presented in Fig. 7. It is observed that a significant degree of variability is present in measured $F_{\mathrm{f}}$ values from one probe to another, with Probe 2 generally exhibiting the highest friction. To eliminate the effect of using different probes on a rational evaluation of the lubrication properties of CVD-grown graphene, ratios of friction coefficients measured for CVD-grown graphene and $\mathrm{SiO}_{2}$ substrates $\left(\mu_{\mathrm{Graphene}} / \mu_{\mathrm{SiO}_{2}}\right)$ have been determined for each probe and consequently averaged to obtain figures of merit for lubrication in Sec. III. B. 5. 


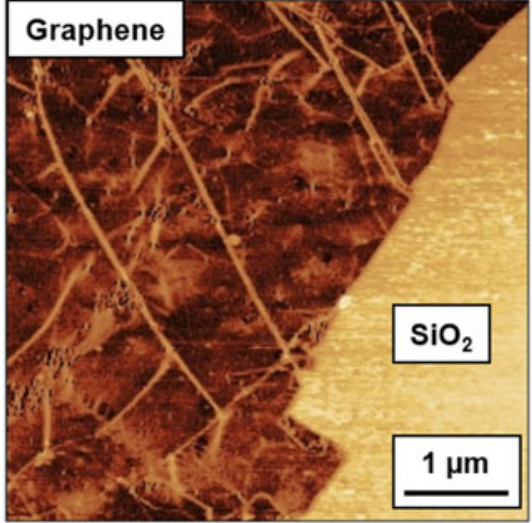

(a)

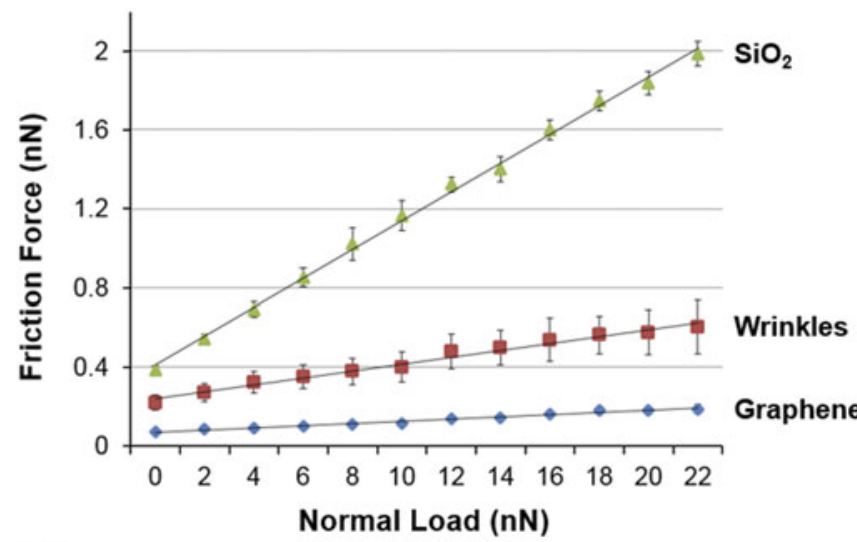

(b)

FIG. 6. (a) Representative friction force map of CVD-grown graphene on $\mathrm{SiO}_{2}$. (b) The dependence of friction force on normal load for graphene, wrinkles, and the $\mathrm{SiO}_{2}$ substrate, together with linear fits. The error bars for measurements on graphene and $\mathrm{SiO}_{2}$ have been determined by considering the standard deviation in friction forces measured on multiple regions of a given friction force map, while the error bars for the measurements on wrinkles have been determined by considering friction forces measured on 10 individual wrinkles. Data acquired with a single probe.

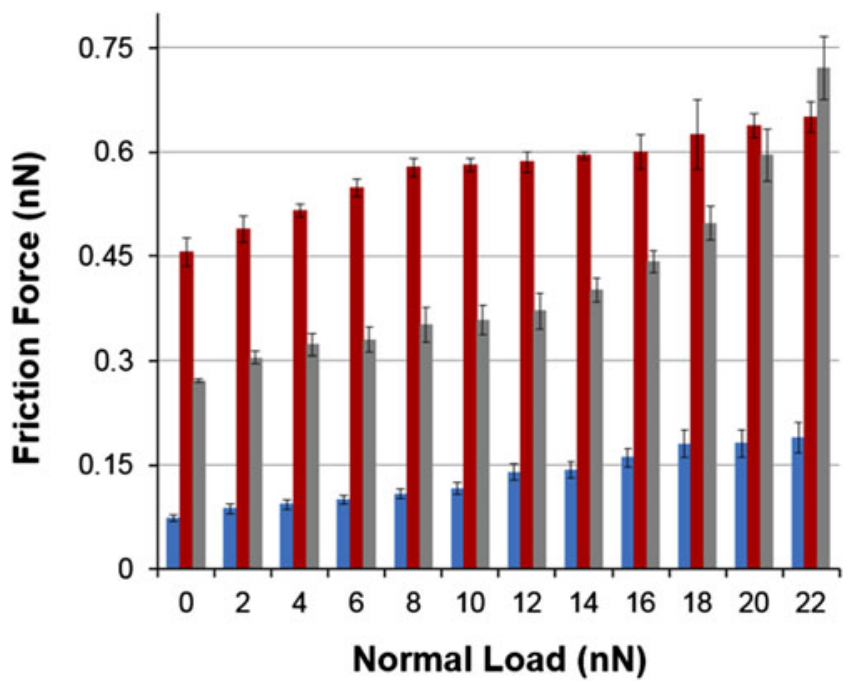

FIG. 7. The dependence of friction force on normal load on CVDgrown graphene for three different probes (Probe 1: blue, Probe 2: red, and Probe 3: gray).

It should be noted that certain degradations associated with tip apexes may occur during the measurements, sometimes resulting in appreciable enlargement of the apex radius and/or contamination by, e.g., post-transfer chemical residues on the sample surface. For instance, the significant increase in $F_{\mathrm{f}}$ observed for Probe 3 in Fig. 7 after $18 \mathrm{nN}$ may be attributed to such a structural/ chemical change. To evaluate whether significant degradation of probe apexes has occurred during the AFM measurements, SEM images of selected probe apexes have been acquired before and after each experiment. It has been observed that the apex radii have increased from 50 to $70 \mathrm{~nm}$, from 25 to $45 \mathrm{~nm}$, and from 55 to $65 \mathrm{~nm}$ for probes $1-3$, respectively. A comparison describing a particularly severe degradation involving Probe 3 is depicted in Fig. 8. Such degradations of sliders are expected to also occur during potential practical implementations of CVD-grown graphene as a solid lubricant. The approach involving the calculation of $\mu_{\mathrm{Graphene}} / \mu_{\mathrm{SiO}_{2}}$ ratios discussed above allows a meaningful evaluation of lubrication performance under such conditions, as well.

\section{Effect of different post-transfer cleaning procedures on friction}

Two post-transfer cleaning procedures have been optionally applied on CVD-grown graphene samples transferred onto $\mathrm{SiO}_{2}$ substrates: (i) ultrasonic baths in acetone and isopropanol and (ii) Ar-gas annealing. Subsequently, the effect of post-transfer cleaning on the tribological behavior of CVD-grown graphene has been investigated (Fig. 9). It is observed that the Ar-gascleaned sample exhibits the highest absolute friction force values, followed by the ultrasonic-cleaned sample, with the as-transferred graphene sample exhibiting the lowest friction forces. It should be indicated that, from this comparison alone, it is not possible to determine the origin of the increase in absolute friction values as the cleaning procedure itself, and not structural/chemical changes in the probe apex that may occur during the experiments. To be able to make rational conclusions regarding this aspect, the previously mentioned approach involving the calculation of $\mu_{\mathrm{Graphene}} / \mu_{\mathrm{SiO}_{2}}$ ratios for multiple probes needs to be used.

\section{Comparison with friction force measurements of mechanically-exfoliated graphene}

While too small for most practical applications, the nanotribological properties of mechanically-exfoliated graphene have been nevertheless the subject of certain 


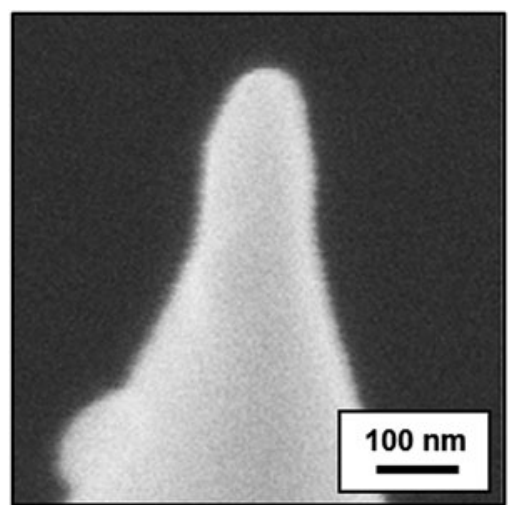

(a)

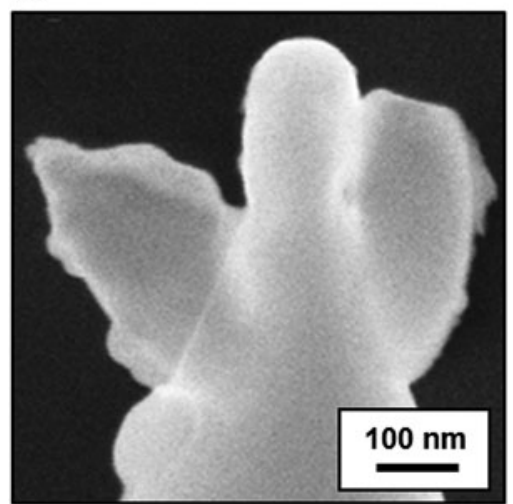

(b)

FIG. 8. SEM images of a specific probe apex (Probe 3) (a) before and (b) after AFM measurements, demonstrating significant degradation.

fundamental studies in the literature. ${ }^{8}$ To determine whether there are significant differences in frictional behavior between single-layer, mechanically-exfoliated and single-layer, CVD-grown graphene, friction force maps have been recorded on both types of samples and comparative measurements of $F_{\mathrm{f}}$ versus $F_{\mathrm{n}}$ have been conducted (Fig. 10). The absence of wrinkles on the mechanically-exfoliated samples is noticeable. While absolute friction force values look very similar for the two graphene samples until a normal load of $18 \mathrm{nN}$ (at which point the specific probe used in the measurements might have undergone a structural/chemical change, as discussed earlier), a thorough comparison of lubrication performance involving $\mu_{\text {Graphene }} / \mu_{\mathrm{SiO}_{2}}$ ratios and multiple probes is provided in Sec. III. B. 5.

\section{Lubrication performance of CVD-grown graphene on $\mathrm{SiO}_{2}$ substrates}

Rather than comparing absolute friction force values, it is useful to employ friction coefficients (extracted from linear fits to $F_{\mathrm{f}}$ versus $F_{\mathrm{n}}$ data) in evaluating graphene's tribological properties, to eliminate the influence of differences in adhesion behavior between graphene and $\mathrm{SiO}_{2}$ substrates. Additionally, to eliminate the effect of (i)

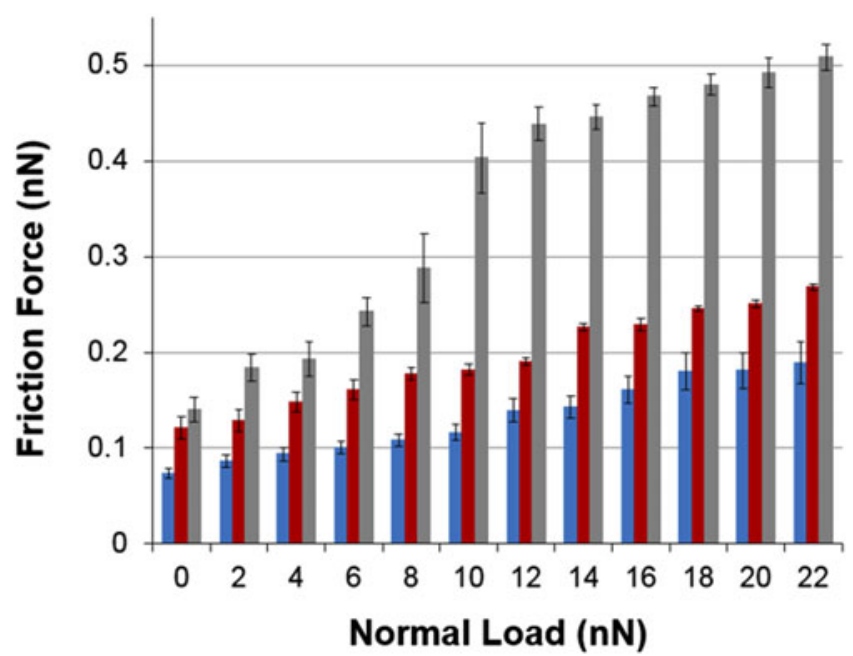

FIG. 9. The dependence of friction force on normal load on CVDgrown graphene for as-transferred (blue), ultrasonic-cleaned (red), and Ar-gas-cleaned (gray) graphene samples. Data acquired with a single probe.

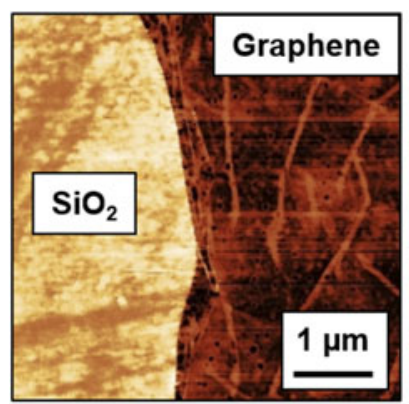

(a)

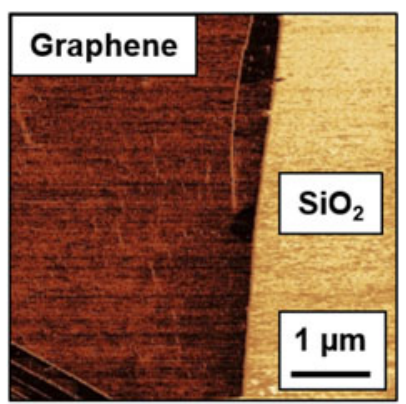

(b)

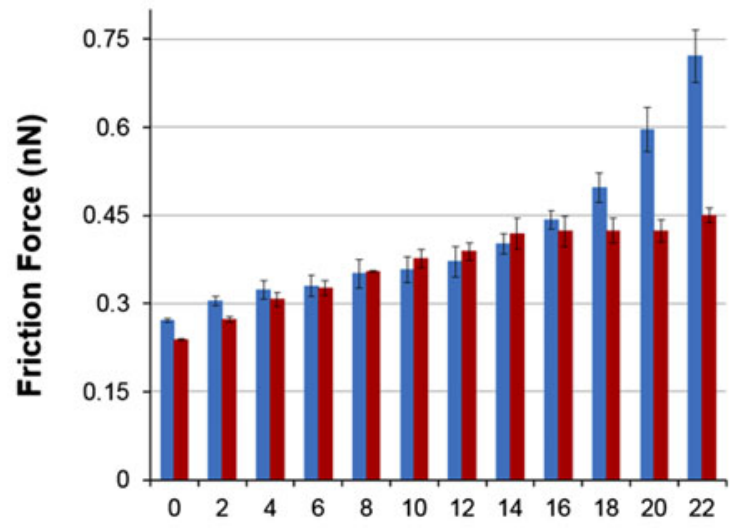

(c)

Normal Load ( $\mathrm{nN})$

FIG. 10. Representative friction force maps for (a) CVD-grown and (b) mechanically-exfoliated graphene samples. (c) The dependence of friction force on normal load for CVD-grown (blue) and mechanicallyexfoliated (red) graphene. Data acquired with a single probe.

using AFM probes with different apex structure/chemistry, and (ii) potential changes in probe apex structure/ chemistry during the experiments, it makes sense to determine ratios of friction coefficients between graphene 
TABLE II. Friction coefficient ratios associated with graphene and $\mathrm{SiO}_{2}\left(\mu_{\text {Graphene }} / \mu_{\mathrm{SiO}_{2}}\right)$.

\begin{tabular}{|c|c|c|c|c|c|c|c|}
\hline Graphene sample & Probe 1 & Probe 2 & Probe 3 & Probe 4 & Probe 5 & Mean & SD \\
\hline As-transferred & $0.076 \pm 0.008$ & $0.042 \pm 0.008$ & $0.103 \pm 0.015$ & $0.078 \pm 0.024$ & - & 0.075 & 0.025 \\
\hline Ultrasonic-cleaned & $0.061 \pm 0.003$ & $0.068 \pm 0.003$ & - & $0.080 \pm 0.051$ & $0.106 \pm 0.042$ & 0.079 & 0.020 \\
\hline Ar-gas-cleaned & $0.124 \pm 0.012$ & - & $0.110 \pm 0.021$ & $0.123 \pm 0.031$ & - & 0.119 & 0.008 \\
\hline Mechanically-exfoliated & - & $0.110 \pm 0.006$ & $0.059 \pm 0.009$ & $0.072 \pm 0.007$ & $0.149 \pm 0.008$ & 0.098 & 0.041 \\
\hline
\end{tabular}

TABLE III. The ratios of friction forces measured on wrinkles to those on surrounding graphene $\left(F_{\mathrm{f} \text {,wrinkle }} / F_{\mathrm{f}, \text { graphene }}\right)$. Data averaged over 10 wrinkles and a normal load range of $0-22 \mathrm{nN}$ for each measurement.

\begin{tabular}{lccccrr}
\hline \hline Graphene sample & Probe 1 & Probe 2 & Probe 3 & Probe 4 & Probe 5 & Mean \\
\hline As-transferred & $3.814 \pm 0.195$ & $3.482 \pm 0.592$ & $2.569 \pm 0.365$ & $3.372 \pm 0.432$ & - & 3.309 \\
Ultrasonic-cleaned & $2.757 \pm 0.083$ & $2.796 \pm 0.156$ & - & $4.017 \pm 1.370$ & $4.018 \pm 0.954$ & 3.397 \\
Ar-gas-cleaned & $2.313 \pm 0.023$ & - & $2.892 \pm 0.314$ & $2.467 \pm 0.116$ & - & 0.717 \\
\hline \hline
\end{tabular}

and the substrate $\left(\mu_{\text {Graphene }} / \mu_{\mathrm{SiO}_{2}}\right)$ for each measurement, and obtain average values from results delivered by the different tips. Mean $\mu_{\text {Graphene }} / \mu_{\mathrm{SiO}_{2}}$ values obtained in this fashion represent true figures of merit associated with the lubricative properties of graphene samples.

Based on the discussion above, $\mu_{\text {Graphene }} / \mu_{\mathrm{SiO}_{2}}$ ratios for CVD-grown graphene in three variants (astransferred, ultrasonic-cleaned, and Ar-gas-cleaned) as well as mechanically-exfoliated graphene are provided in Table II, for (up to) five different probes, together with mean and standard deviation values. By studying the data, it is seen that graphene in all examined forms acts as a very good solid lubricant, reducing friction coefficients observed on $\mathrm{SiO}_{2}$ substrates by $\sim 90 \%$. Among CVDgrown graphene samples, the as-transferred variety exhibits the highest mean lubrication performance (thus, the lowest $\mu_{\text {Graphene }} / \mu_{\mathrm{SiO}_{2}}$ ratio) and the mean lubrication performance drops slightly with ultrasonic cleaning, while Ar-gas-cleaned samples exhibit the lowest mean lubrication performance. It should be mentioned that a consideration of standard deviation values together with the mean values reported for as-transferred and ultrasonic-cleaned samples indicates that there is no statistically-significant difference in overall lubrication performance between the two varieties of graphene. On the other hand, Ar-gas-cleaned samples clearly exhibit a comparatively lower lubrication performance. A potential physical mechanism responsible for this effect could involve the thermal breakdown of PMMA residue trapped under the transferred graphene samples during annealing, leading to a wide distribution of trapped contaminants increasing average surface roughness and friction. Finally, it should be noted that while Raman spectroscopy has been previously used to successfully correlate the degree of fluorination of graphene samples to changes in frictional behavior, ${ }^{33}$ no direct correlation between the "quality" of graphene samples (as inferred from $I_{2 \mathrm{D} / \mathrm{G}}$ values) and lubrication performance has been observed in our studies.

Our results also allow a comparison of the lubrication performance of CVD-grown and mechanically-exfoliated graphene samples. While it is seen that as-transferred CVD-grown graphene samples on average exhibit slightly better lubrication performance than exfoliated ones, the existence of wrinkles on CVD-grown graphene and the associated increase in friction should be taken into account (please note that the data for CVD-grown graphene presented in Table II have been acquired on regions free from wrinkles). In that respect, the extent to which wrinkles increase friction values on CVD-grown graphene (as-transferred, ultrasonic-cleaned, and Ar-gascleaned) is examined in Table III. As expected, wrinkles lead to a significant (about 3-fold) enhancement of local friction values on CVD-grown graphene samples, representing a considerable detriment to overall lubrication performance.

\section{CONCLUSIONS}

A comprehensive characterization of nanoscale structural and tribological properties of CVD-grown, singlelayer graphene transferred onto $\mathrm{SiO}_{2}$ substrates has been presented. To analyze lubrication performance, an approach involving the evaluation of friction coefficient ratios of graphene and $\mathrm{SiO}_{2}$ has been used. The effect of using different probes and employing different posttransfer cleaning procedures on frictional behavior has been studied. Results have shown that CVD-grown graphene acts as an effective solid lubricant on $\mathrm{SiO}_{2}$, leading to a reduction of $\sim 90 \%$ in the friction coefficients observed via AFM. While no significant difference in lubrication behavior has been found on wrinkle-free regions of CVD-grown graphene when compared to mechanically-exfoliated samples, it has been 
shown that wrinkles on CVD-grown graphene lead to a substantial (about 3-fold) increase in recorded friction values.

\section{ACKNOWLEDGMENTS}

The authors would like to thank Arda Balkancı, Kinyas Polat and Şahin Beşerik for practical help with various aspects of the experiments presented in this work. Financial support from the Outstanding Young Scientist Program of the Turkish Academy of Sciences (TÜBA-GEBIP) is gratefully acknowledged.

\section{REFERENCES}

1. M. Urbakh, J. Klafter, D. Gourdon, and J. Israelachvili: The nonlinear nature of friction. Nature 430, 525 (2004).

2. S.M. Hsu: Nano-lubrication: Concept and design. Tribol. Int. 37, 537 (2004)

3. K.S. Novoselov, A.K. Geim, S.V. Morozov, D. Jiang, Y. Zhang, S.V. Dubonos, I.V. Grigorieva, and A.A. Firsov: Electric field effect in atomically thin carbon films. Science 306, 666 (2004).

4. C. Lee, X. Wei, J.W. Kysar, and J. Hone: Measurement of the elastic properties and intrinsic strength of monolayer graphene. Science 321, 385 (2008).

5. D. Berman, A. Erdemir, and A.V. Sumant: Graphene: A new emerging lubricant. Mater. Today 17, 31 (2014).

6. T. Filleter, J.L. McChesney, A. Bostwick, E. Rotenberg, K.V. Emtsev, T. Seyller, K. Horn, and R. Bennewitz: Friction and dissipation in epitaxial graphene films. Phys. Rev. Lett. 102, 086102 (2009).

7. T. Filleter and R. Bennewitz: Structural and frictional properties of graphene films on $\mathrm{SiC}(0001)$ studied by atomic force microscopy. Phys. Rev. B 81, 155412 (2010).

8. C. Lee, Q. Li, W. Kalb, X.Z. Liu, H. Berger, R.W. Carpick, and J. Hone: Frictional characteristics of atomically thin sheets. Science 328, 76 (2010).

9. K-S. Kim, H-J. Lee, C. Lee, S-K. Lee, H. Jang, J-H. Ahn, J-H. Kim, and H-J. Lee: Chemical vapor deposition-grown graphene: The thinnest solid lubricant. ACS Nano 5, 5107 (2011).

10. Y.J. Shin, R. Stromberg, R. Nay, H. Huang, A.T.S. Wee, H. Yang, and C.S. Bhatia: Frictional characteristics of exfoliated and epitaxial graphene. Carbon 49, 4070 (2011).

11. G. Fessler, B. Eren, U. Gysin, T. Glatzel, and E. Meyer: Friction force microscopy studies on $\mathrm{SiO}_{2}$ supported pristine and hydrogenated graphene. Appl. Phys. Lett. 104, 041910 (2014).

12. P. Egberts, G.H. Han, X.Z. Liu, A.T.C. Johnson, and R.W. Carpick: Frictional behavior of atomically thin sheets: Hexagonal-shaped graphene islands grown on copper by chemical vapor deposition. ACS Nano 8, 5010 (2014).

13. G. Paolicelli, M. Tripathi, V. Corradini, A. Candini, and S. Valeri: Nanoscale frictional behavior of graphene on $\mathrm{SiO}_{2}$ and $\mathrm{Ni}(111)$ substrates. Nanotechnology 26, 055703 (2015).

14. A. Reina, X. Jia, J. Ho, D. Nezich, H. Son, V. Bulovic, M.S. Dresselhaus, and J. Kong: Large area, few-layer graphene films on arbitrary substrates by chemical vapor deposition. Nano Lett. 9, 3087 (2009).

15. X. Li, W. Cai, J. An, S. Kim, J. Nah, D. Yang, R. Piner, A. Velamakanni, I. Jung, E. Tutuc, S.K. Banerjee, L. Colombo, and R.S. Ruoff: Large-area synthesis of high-quality and uniform graphene films on copper foils. Science 324, 1312 (2009).
16. M. Her, R. Beams, and L. Novotny: Graphene transfer with reduced residue. Phys. Lett. A 377, 1455 (2013).

17. A. Pirkle, J. Chan, A. Venugopal, D. Hinojos, C.W. Magnuson, S. McDonnell, L. Colombo, E.M. Vogel, R.S. Ruoff, and R.M. Wallace: The effect of chemical residues on the physical and electrical properties of chemical vapor deposited graphene transferred to $\mathrm{SiO}_{2}$. Appl. Phys. Lett. 99, 122108 (2011).

18. B. Hu, Z. Wei, H. Ago, Y. Jin, M. Xia, Z. Luo, Q. Pan, and Y. Liu: Effects of substrate and transfer on CVD-grown graphene over sapphire-induced Cu films. Sci. China: Chem. 57, 895 (2014).

19. Y. Dan, Y. Lu, N.J. Kybert, Z. Luo, and A.T.C. Johnson: Intrinsic response of graphene vapor sensors. Nano Lett. 9, 1472 (2009).

20. C. Gong, H.C. Floresca, D. Hinojos, S. McDonnell, X. Qin, Y. Hao, S. Jandhyala, G. Mordi, J. Kim, L. Colombo, R.S. Ruoff, M.J. Kim, K. Cho, R.M. Wallace, and Y.J. Chabal: Rapid selective etching of PMMA residues from transferred graphene by carbon dioxide. J. Phys. Chem. C 117, 23000 (2013).

21. C.W. Jang, J.H. Kim, J.M. Kim, D.H. Shin, S. Kim, and S-H. Choi: Rapid-thermal-annealing surface treatment for restoring the intrinsic properties of graphene field-effect transistors. Nanotechnology 24, 405301 (2013).

22. V. Skakalova and A.B. Kaiser: Graphene: Properties, Preparation, Characterisation and Devices, 1st ed. (Woodhead Publishing, Cambridge, England, 2014); pp. 279-287.

23. A.C. Ferrari, J.C. Meyer, V. Scardaci, C. Casiraghi, M. Lazzeri, F. Mauri, S. Piscanec, D. Jiang, K.S. Novoselov, S. Roth, and A.K. Geim: Raman spectrum of graphene and graphene layers. Phys. Rev. Lett. 97, 187401 (2006).

24. C.M. Mate, G.M. McClelland, R. Erlandsson, and S. Chiang: Atomic-scale friction of a tungsten tip on a graphite surface. Phys. Rev. Lett. 59, 1942 (1987).

25. J.E. Sader, J.W.M. Chon, and P. Mulvaney: Calibration of rectangular atomic force microscope cantilevers. Rev. Sci. Instrum. 70, 3967 (1999).

26. M. Varenberg, I. Etsion, and G. Halperin: An improved wedge calibration method for lateral force in atomic force microscopy. Rev. Sci. Instrum. 74, 3362 (2003).

27. U.D. Schwarz, P. Koster, and R. Wiesendanger: Quantitative analysis of lateral force microscopy experiments. Rev. Sci. Instrum. 67, 2560 (1996).

28. K. Celebi: Chemical Vapor Deposition of Graphene on Copper (ETH Zurich, Zurich, 2013); p. 24.

29. S. Berciaud, S. Ryu, L.E. Brus, and T.F. Heinz: Probing the intrinsic properties of exfoliated graphene: Raman spectroscopy of free-standing monolayers. Nano Lett. 9, 346 (2009).

30. T.F. Chung, T. Shen, H. Cao, L.A. Jauregui, W. Wu, Q. Yu, D. Newell, and Y.P. Chen: Synthetic graphene grown by chemical vapor deposition on copper foils. Int. J. Mod. Phys. B 27, 1341002 (2013).

31. S. Sundararajan and B. Bhushan: Topography-induced contributions to friction forces measured using an atomic force/friction force microscope. J. Appl. Phys. 88, 4825 (2000).

32. I. Szlufarska, M. Chandross, and R.W. Carpick: Recent advances in single-asperity nanotribology. J. Phys. D: Appl. Phys. 41, 123001 (2008).

33. Q. Li, X-Z. Liu, S-P. Kim, V.B. Shenoy, P.E. Sheehan, J.T. Robinson, and R.W. Carpick: Fluorination of graphene enhances friction due to increased corrugation. Nano Lett. 14, 5212 (2014). 